\title{
LECTURA Y TRADUCCIÓN LITERARIA: DESAFÍOS EN LA FORMACIÓN DE TRADUCTORES
}

\author{
READING PROCESSES AND LITERARY TRANSLATION: THE CHALLENGES FACED BY \\ STUDENTS OF TRANSLATION
}

Dolores Aicega $^{1}$

\begin{abstract}
RESUMEN: El objetivo general de esta investigación es explorar los distintos desafíos que presenta la lectura para la traducción literaria a los estudiantes del Traductorado Público de una universidad nacional argentina. Para ello, se consideraron aportes de los estudios sobre la comprensión de textos y de la traductología, y se incorporó la perspectiva bajtiniana para abordar los textos y la traducción literaria. Se realizó un estudio exploratorio, cualitativo y transversal del que participaron 55 estudiantes del cuarto año de una universidad nacional argentina y se analizaron 55 traducciones que tales estudiantes produjeron en el marco de la cursada de Traducción Literaria 1. Los resultados obtenidos mediante el análisis de este corpus permiten concluir que la lectura como parte de la traducción literaria impone a los futuros traductores desafíos que se relacionan, por un lado, con la elaboración de los distintos niveles de representación de la información textual: estructura superficial, base del texto y modelo de situación. Por otra parte, la interpretación del texto fuente como artefacto lingüístico, sociocultural y literario que incorpora informaciones de diverso tipo genera desafíos adicionales a los estudiantes. Finalmente, estos desafíos dan cuenta de una competencia traductora aún en formación.
\end{abstract}

Palabras clave: Traducción literaria; lectura; formación de traductores.

ABSTRACT: This paper aims to explore the processes involved in reading literary texts as part of the translation process. More specifically, we seek to identify the challenges faced by undergraduate students of translation (Spanish-English) in an Argentine public university when they have to read and translate literary texts in the context of their studies. Our theoretical framework brings together developments from the fields of text comprehension studies and translation studies. Our research involved the analysis of 55 translations carried out by students of Literary Translation 1 as part of their practice. Working with this corpus has allowed us to establish that the reading processes involved in literary translation impose on the students challenges which are associated with: a) the elaboration of the different levels of representation of the textual information: surface structure, text base and situation model; b) the interpretation of the source text as a linguistic, sociocultural and literary artifact which incorporates diverse information. The challenges we have identified are also related to the emergence of a translation competence which is beginning to develop.

Key words: literary translation; reading processes, teaching translation

\footnotetext{
${ }^{1}$ Magister en Psicología Cognitiva y Aprendizaje de FLACSO. Profesora en Lengua y Literatura Inglesas de la Universidad Nacional de La Plata- UNLP. Centro de Estudios de Literatura y Literaturas Comparadas (CELYLC) Instituto de Instituto de Investigaciones en Humanidades y Ciencias Sociales (IDHICS) Facultad de Humanidades y Cs. de la Educación (FaHCE), Universidad Nacional de La Plata (UNLP).
} 


\section{Introducción}

La traducción, entendida como proceso, puede concebirse en atención a tres etapas o procedimientos principales: la comprensión del texto original o fuente, generalmente acompañada de un análisis de la especialidad funcional del texto por traducir, la reformulación de ese texto en la lengua meta y la revisión o corrección del texto traducido, es decir, del texto meta. En este sentido, varias investigaciones han descripto la tarea traductológica en relación con tres etapas o fases normalmente definidas como la fase previa a la escritura del texto meta, la fase en la que se escribe el texto meta y la fase posterior a la escritura del texto meta (VázquezAyora, 1977; Nida, 1969; Krings 1986a, Jääskeläinen 1999, Norberg 2003, Jakobsen 2003). Con una mirada centrada en los procesos de escritura, Jääskeläinen (1999) distingue tres fases del proceso traductor a las que denomina pre-escritura, escritura y post-escritura. Englund Dimitrova (2005), por su parte, concibe el proceso en términos de tres procesos principales: planificación (dentro de la cual se ubica la lectura del TF), generación del texto, y revisión.

Distintas investigaciones (Ayupova, 2013; Belova, 2015; Nord, 1991) conciben la primera etapa del proceso traductor como un análisis del texto fuente (en adelante, TF) previo a la traducción del mismo. El objetivo de este análisis consiste en lograr una comprensión acabada del TF que posibilite una traducción que resulte apropiada en función del contexto en el que circulará la misma. Así, por ejemplo, Belova (2015) caracteriza esta etapa de la traducción como un análisis organizado que permite al traductor hacer del TF un fenómeno más "observable” en términos de su lenguaje y estructura. Este análisis implica, asimismo, delinear la organización semántica y comunicativa del TF, elaborar todo su significado y sentido de manera pormenorizada, determinar la estrategia de traducción, identificar las estructuras y aspectos característicos del tipo de texto y sus peculiaridades lingüísticas, y establecer el valor informativo de los segmentos del texto, entre otros. Por su parte, Nord (1991) señala la necesidad de elaborar un modelo del análisis del texto fuente que sea diseñado dentro del campo de los estudios de traducción, y responda a las necesidades específicas de los traductores profesionales. El análisis del texto fuente implica, para esta autora, un análisis pormenorizado de las propiedades gramaticales, semánticas, estilísticas del texto fuente que asegure al traductor una comprensión completa de los significados denotativo y connotativo del texto fuente.

Las observaciones de Nord (1991) y Belova (2015) están en línea con un espectro amplio de traductólogos que caracteriza la lectura en la traducción literaria como un tipo de lectura que se distingue de la lectura con otros fines. Según señala Wærn (1979), normalmente el propósito con el que se lee un texto ejerce una influencia en cómo se lee ese texto y qué aspectos del mismo reciben mayor atención. Se desprende, entonces, que la tarea inminente de la traducción condiciona el modo en el que el traductor se enfrenta al TF en la tarea de la lectura, que es, además, una parte integral del proceso traductor. Reiss y Vermeer (1984: 1972) sostienen que durante la lectura del TF, los traductores suelen identificar los pasajes "difíciles" y acostumbran identificar posibles equivalentes de traducción. Esta observación indica que la consciencia respecto de la tarea traductológica ejerce una influencia en los procesos de percepción y comprensión del TF. Shreve y Diamond (1997:246-247) observan que los traductores profesionales no solamente elaboran la estructura lingüística durante la lectura del 
TF, sino que, además, extraen del TF información relevante para la traducción. En esta misma línea, Shreve (1993) analiza la lectura de un mismo texto con tres propósitos distintos: para parafrasearlo, para extraer el contenido proposicional principal, y finalmente, para traducirlo. El estudio revela que los sujetos que leyeron el texto con el propósito de traducirlo, todos ellos estudiantes de primer año de traducción, identificaron más problemas y, en parte, problemas de una naturaleza distinta que los lectores de los otros dos grupos.

En su estudio sobre la traducción de poesía, Díaz-Diocaretz (1985) reconoce el carácter dual del traductor en tanto lector y escritor. Según esta autora, como lector activo, el traductor intentará comprender e interpretar todos los operadores temáticos que permiten recodificar las estructuras formales del texto en significados. La lectura como parte de los procesos implicados en la traducción literaria, por tanto, implica explorar el TF de un modo mucho más exhaustivo que el que lleva adelante el crítico o el lector ordinario. Desde esta perspectiva, Díaz-Diocaretz señala que, además del estilo y lenguaje literarios del autor o poeta, la estructura del texto, su significado y sentido, las elecciones interpretativas del traductor juegan un papel fundamental en los procesos de selectividad. En este sentido, las selecciones del traductor durante las fases de la lectura y de la escritura dan a la obra literaria una nueva identidad.

Por otra parte, en una reflexión acerca de la recepción de las traducciones (???), Lawrence Venuti (2011), uno de los teóricos de la traducción más prestigiosos de la actualidad, sostiene que el lector de una traducción no puede experimentar una respuesta al texto que resulte equivalente o siquiera comparable a la respuesta con la que el lector de la lengua fuente experimenta el TF ya que la respuesta del lector de la lengua fuente se relaciona con la experiencia de estar inmenso en la cultura fuente. Venuti incluso señala que ni siquiera el lector bilingüe, que está familiarizado con las culturas fuente y meta, experimenta los dos textos de un modo similar.

Desde el campo más específico de los estudios psicolingüísticos centrados en la traducción, Bell (1998) reconoce que los procesos de comprensión en comunicadores bilingües y monolingües son diferentes. Si bien tanto unos como otros recogen información de un texto particular, para el comunicador monolingüe, este es el objetivo primordial. El traductor, en cambio, necesita ser capaz de reconocer elementos relevantes del texto que pueden constituir problemas de traducción o que reflejan variables significativas que deben ser plasmadas en el texto meta (en adelante, TM). Por otra parte, Bell señala que los traductores e intérpretes "escuchan y leen" de una manera distinta a otros usuarios del lenguaje porque operan bajo un conjunto de limitaciones diferente. Las limitaciones que enfrenta el traductor están dadas por la tarea (la actividad que tiene que realizar el traductor y el contexto en el que tiene que desarrollarla), el texto (la estructura lingüística y discursiva del TF) y el traductor (las habilidades y conocimientos lingüísticos y no-lingüísticos de la persona que realiza la traducción).

Bell señala, asimismo, que estudiar el modo en que los traductores e intérpretes elaboran la información en procesos que resultan diferentes a los que realizan hablantes y escritores no traductores es un objetivo fundamental de la disciplina. El autor sostiene que, si bien un modelo de traducción replica las características generales de la comunicación humana, el mismo debe incluir aspectos que son específicos de la traducción, particularmente en relación con la identificación y resolución de problemas. Asimismo, los roles de los 
comunicadores monolingües y los traductores como receptores del mensaje también difieren. Los primeros están orientados al emisor y prestan atención al mensaje para responder al mismo mediante el acuerdo, desacuerdo, enojo, entre otros; los traductores, por su parte, están orientados al receptor y prestan atención al mensaje del hablante/escritor para retransmitirlo a los receptores del TM. En su rol de re-transmisores del mensaje, los traductores suprimen, o al menos intentan controlar, sus reacciones personales al mensaje. En efecto, Seleskovitch (1978) muestra que los traductores piensan que deben "disciplinar su entendimiento" para privilegiar las expectativas y suposiciones de los receptores a los que está dirigida la traducción/interpretación.

En cuanto al procesamiento del texto, Bell establece que este comprende, principalmente, la resolución de problemas. Al igual que otros procesadores de texto, los traductores enfrentan problemas de comprensión, interpretación y expresión y desarrollan estrategias para superarlos. En esta misma línea, como señala Krings (1987), el área de los estudios del proceso traductor debe identificar los problemas que enfrentan los traductores y la frecuencia con la que ocurren y las estrategias que se emplean para su reconocimiento y resolución.

Este trabajo se centra en el primer paso del proceso de la traducción literaria, la lectura y comprensión del texto fuente, que, como señalan William y Chesterman (2014) consiste en el análisis cuidadoso de los aspectos potencialmente problemáticos del texto fuente como parte de la preparación para la traducción propiamente dicha. Dancette $(1995)$ y Kussmaul $(1996,1997)$, por su parte, destacan la importancia de esta etapa como un elemento vital para el éxito del proceso traductor. En esta investigación nos proponemos indagar acerca de los desafíos que este tipo particular de lectura impone a los estudiantes de traducción en el ámbito de la universidad pública.

La traducción literaria, objeto de nuestro estudio aquí, es una práctica compleja que supone, además, ciertos conocimientos por parte del traductor que exceden, en ocasiones por mucho, el territorio de lo estrictamente lingüístico. Estos saberes, sin embargo, resultan necesarios para lograr una comprensión y traducción del texto fuente que sea coherente y adecuada en relación tanto con el contexto de producción o fuente como con el contexto de recepción o meta.

Esta investigación se propone abordar los desafíos que presenta la lectura en la traducción literaria a los traductores en formación. Se trata de un tipo de lectura que requiere como contraparte de un lector que sea capaz de enfrentar estos desafíos. En el marco de nuestro trabajo, utilizamos la noción de lector-traductor para designar al lector que cuenta con las habilidades y los saberes necesarios para enfrentar la complejidad de los procesos que ofrece la lectura para la traducción literaria. Por otra parte, en atención a nuestro interés en la formación de traductores, recurrimos al concepto de lector-traductor en formación para referirnos a los estudiantes de traducción que están transitando sus estudios en traducción literaria y se encuentran en proceso de desarrollo de los conocimientos y habilidades aludidos.

Desde una mirada pedagógica, Carlino (2005) hace hincapié en la necesidad de que la universidad enseñe a leer en el interior de las disciplinas, atendiendo a las particularidades que adquieren la lectura y la escritura en cada campo disciplinario. En consonancia con los estudios 
sobre alfabetización académica que surgieron en el contexto anglosajón a mediados de la década de los años noventa, Carlino destaca la importancia central de la lectura en la vida académica universitaria y advierte sobre la tendencia a considerarla una habilidad general básica, que se logra de una vez y para siempre. Por otra parte, la complejidad y diversidad de intereses e inquietudes, propósitos, audiencias, entre otros, exigen continuar aprendiendo a leer y a escribir de manera continua. Asimismo, la investigadora y docente universitaria propone que las cátedras planifiquen estratégicamente la enseñanza de la lectura. En esta investigación adherimos a este postulado y buscamos contribuir a dar cuenta de la especificidad de los procedimientos que implica la lecto-comprensión para la traducción literaria en el ámbito de la formación universitaria y los desafíos de enseñanza y aprendizaje que ella conlleva.

\section{Aspectos metodológicos}

El objetivo general de nuestro trabajo consiste en identificar los desafíos que enfrentan los estudiantes de la carrera de Traductorado Público en Lengua Inglesa de una universidad nacional a la hora de leer textos literarios para su traducción. Para ello, utilizamos un marco teórico que incorpora desarrollos provenientes de los estudios sobre la comprensión del discurso y de la traductología o estudios de traducción. Por un lado, el modelo de van Dijk y Kintsch (van Dijk, 1978; 1980; 2014; van Dijk y Kintsch 1983; Kintsch, 1988 y 1998) aporta una teoría ampliamente aceptada de la comprensión textual que nos permite desentrañar los procesos implicados en la lectura de textos. En relación con esta teoría, haremos hincapié en los conceptos de macroestructura y superestructura y en los distintos niveles de representación del significado de un texto: a) la estructura superficial que compromete las características lingüísticas y gramaticales del texto; b) la representación proposicional, o base del texto, que hace referencia a las ideas explícitas del texto y las relaciones semánticas que se establecen entre ellas; c) el modelo de situación, que consiste en una representación mental del texto en la que se integran las proposiciones del texto con el conocimiento del lector. Por otro lado, utilizamos los conceptos de mapa del texto y planos de Holmes ([1978]1988) a fin de caracterizar los procesos de lectura que intervienen en el proceso de traducción. Holmes sostiene que la traducción se efectúa en dos planos: un plano serial, en el que la traducción se realiza palabra por palabra, oración por oración; y un plano estructural en el que se extrae una concepción mental de texto fuente en su totalidad. Esta concepción mental o mapa del texto fuente constituye un criterio general en la formulación del texto meta. De manera simultánea, el traductor se va formando una imagen del mapa del texto meta, la cual guiará la escritura de su texto. Por último, recurriremos a la noción de competencia traductora de Hurtado Albir (1996, 2001) y el grupo PACTE (2003) para describir los saberes y competencias que los traductores en formación deben desarrollar a fin de poder llevar adelante la tarea traductológica con éxito.

Para indagar sobre los desafíos que presenta la lectura de textos literarios al lectortraductor en formación, hemos analizado producciones de los estudiantes de Traducción Literaria 1 de la carrera de Traductorado Público Nacional en Lengua Inglesa de una universidad nacional argentina. Nuestro corpus consiste en cincuenta y cinco traducciones de fragmentos de textos literarios narrativos realizadas en clase como prácticas de traducción. Las 
traducciones que hemos seleccionado y analizado corresponden a un fragmentos del cuento "Dead Men's Path" de Chinua Achebe.

Por medio de nuestra investigación se intentará dar respuesta al interrogante: ¿Cuáles son los desafíos que presenta la lectura para la traducción literaria a los estudiantes del Traductorado Público Nacional de una universidad nacional argentina?

Para ello, resulta necesario definir la extensión que tienen los términos clave en el marco de nuestra investigación. En el marco de este trabajo, el término "desafíos" hace referencia a los retos, obstáculos o escollos que deben enfrentar los estudiantes de traducción literaria en la etapa de la lectura. Entendemos la lectura como el análisis del TF previo a su traducción en lo que comúnmente se conoce como la primera etapa del proceso traductor (Vázquez-Ayora, 1977; Nida, 1969; Krings 1986a, Jääskeläinen 1999, Norberg 2003, Jakobsen 2003). Por último, sin desconocer los debates alrededor de la naturaleza de la traducción literaria, la misma es entendida en esta investigación como la traducción de originales "literarios" en la que se espera que los traductores preserven o recreen de algún modo las intenciones o efectos estéticos del original (Delabastita, 2011).

Más específicamente, a lo largo de la investigación, nos proponemos dar respuesta a los siguientes interrogantes:

- ¿Cuáles son los desafíos particulares que enfrentan los estudiantes de Traducción Literaria 1 en la elaboración de los distintos niveles de representación del texto fuente: nivel superficial, base del texto y modelo de situación?

- ¿Cuáles son los retos de interpretación que presenta el TF en sus dimensiones de artefacto lingüístico, literario y sociocultural para los traductores en formación?

- ¿Qué pueden decirnos estos desafíos de comprensión acerca del desarrollo de la competencia traductora de los estudiantes?

\subsection{Objetivos}

Objetivo general:

- Explorar los desafíos que presenta la lectura para la traducción literaria a los estudiantes del Traductorado Público Nacional de una universidad nacional.

Objetivos específicos:

- Identificar los desafíos de lectura en relación con la elaboración de la estructura superficial, la base textual y el modelo de situación del texto literario en tanto TF.

- Conocer las dificultades que enfrentan los estudiantes al interpretar el TF en sus dimensiones lingüística, literaria y sociocultural.

- Indagar sobre el desarrollo de la competencia traductora de los estudiantes a la luz de los desafíos que presentan las traducciones.

\subsection{Método}

Hemos realizado un estudio exploratorio-transversal. Siguiendo a Hernández Samperi, 
Fernández Collado y Baptista Lucio (1991), los estudios transversales o transeccionales, son aquellos que analizan un fenómeno en un momento dado, a diferencia de los estudios longitudinales, que lo hacen a través del tiempo. Por otra parte, el estudio exploratorio permite indagar sobre problemas de investigación poco estudiados con vistas a identificar conceptos o variables promisorias, establecer prioridades y sugerir postulados para futuras investigaciones.

\subsection{Muestra}

La muestra fue seleccionada de manera intencional no probabilística. El criterio de selección, que fue establecido por la investigadora con anterioridad a la investigación, y estableció que los participantes fueran alumnos de Traducción Literaria 1 en una carrera de Traductorado Público de una universidad nacional. Se reunieron 55 participantes cuya media de edad fue de 23 años.

\subsection{Instrumentos y procedimientos}

Como instrumento para la recolección de datos utilizamos traducciones realizadas por los estudiantes en el marco de la cursada de la materia Traducción Literaria 1. Según consta en el programa de la asignatura, la modalidad de trabajo en Traducción Literaria 1 consiste en la lectura minuciosa de distintos textos literarios dramáticos y narrativos (cuentos, fragmentos de novelas y de textos dramáticos) y de textos expositivos (ensayos, artículos críticos) por parte de los estudiantes y la discusión en clase de distintos aspectos de los mismos desde la perspectiva de un potencial traductor de estos textos. Como parte de la práctica que se desarrolla en la clase, los estudiantes deben resolver distintas tareas de traducción a lo largo del año que, normalmente, incluyen la traducción del inglés al español de fragmentos de cuentos, ensayos y artículos críticos discutidos previamente en clase.

En nuestra investigación hemos analizado la producción de los estudiantes en tareas de práctica de traducción cuyo texto fuente fue un fragmento del cuento "Dead Men's Path" de Chinua Achebe. El criterio de selección del corpus responde al interés específico de la traducción literaria en esta área de la traductología. Por el contrario, los ensayos y artículos críticos que se trabajan en la cátedra de Traducción Literaria 1 pueden ser abordados desde otras áreas de los estudios de traducción (traducción científica, técnica, de textos académicos), cuyo objeto de estudio son los textos comúnmente denominados "pragmáticos” (Delisle, 1985).

Se analizaron cincuenta y cinco traducciones realizadas por los estudiantes en clase como prácticas de traducción. El texto fuente es un "texto conocido" (analizado y comentado previamente en clase). Los estudiantes cuentan con una hora para realizar la tarea y tienen acceso libre a materiales de referencia (diccionarios monolingüe y bilingüe, enciclopedias, manuales). El análisis de estas traducciones nos aporta pistas valiosas sobre las ideas que se forman los estudiantes de los textos literarios por traducir. Aunque de manera indirecta, las traducciones nos permiten reflexionar sobre los desafíos la lectura y la comprensión de textos literarios para los traductores en formación. En este sentido, en nuestro análisis hemos seleccionado los casos o ejemplos de las traducciones de los estudiantes que se relacionan con 
problemas de comprensión y hemos hecho a un lado aquellos que se vinculan con la expresión de los significados en la lengua meta (español).

Hemos utilizado el método de comparación continua para analizar nuestros corpus de traducciones. Esta metodología de análisis permite la comparación de incidentes específicos de los datos a fin de desarrollar conceptos, identificar sus propiedades y explorar sus interrelaciones. A través de esta metodología, hemos analizado una variedad de procesos, conocimientos y habilidades que están implicados en la lectura de textos literarios como parte de la tarea traductológica. Nuestro análisis contempló las nociones de macroestructura y superestructura y los tres niveles de procesamiento de la información que describe el modelo de van Dijk y Kintsch (van Dijk, 1978; 1980; 2014; van Dijk y Kintsch 1983; Kintsch, 1988 y 1998): la estructura superficial (las propiedades lingüísticas y gramaticales del texto), la base del texto (la secuencia de proposiciones expresada por la secuencia de oraciones del texto) y el modelo de situación (la representación mental de los textos escritos y orales que combina la información del texto con los conocimientos del lector). Esta división tiene como fin ordenar y organizar la presentación del análisis de las traducciones ya que, en la práctica, los tres niveles operan de manera concomitante en los procesos de comprensión textual. Asimismo, hemos analizado el corpus en relación con los tipos de información que aporta el texto (contextual, intertextual y situacional) según el modelo de Holmes, ([1978]1988); esta parte del análisis nos permitió indagar sobre las dificultades que presenta la interpretación de textos literarios en sus dimensiones literaria y sociocultural. Por último, nuestro análisis incorporó el concepto de competencia traductora de Hurtado Albir (1996, 2001) y el grupo PACTE (2003) para comprender mejor las habilidades y competencias que requiere la tarea de comprensión de estos textos en tareas de traducción.

El primer paso de la investigación consistió en la familiarización de la investigadora con el ámbito de la cátedra a la que pertenecen los estudiantes de la investigación. Esta etapa incluyó conversaciones y comunicaciones con los docentes de la cátedra y los estudiantes, familiarización con el plan de estudios de la carrera y programa de la asignatura, lectura de los materiales de clase y estrategias de evaluación, entre otros.

La segunda etapa fue la selección del corpus. La cátedra puso a nuestra disposición las traducciones de los estudiantes de cuatro textos narrativos: a) "Dead Men's Path" de Chinua Achebe; b) "The Flying Stars" de Chesterton; c) I know why the caged bird sings de M. Angelou; y d) "The Cat the Walked by Himself" de R. Kipling. Luego de leer y hacer un análisis preliminar de todas las traducciones, que sumaban un total de 231 textos, se decidió focalizar la investigación en las traducciones de "Dead Men’s Path”. La razón principal de esta decisión fue mantener el corpus en un tamaño acorde a las características, tiempos y recursos de la investigación. Por otra parte, las traducciones del fragmento de I know why the caged bird sings fueron descartadas porque, a diferencia de las traducciones restantes, en ese caso los estudiantes no habían leído el texto completo. En consecuencia, parte de los problemas de comprensión que encontramos en las traducciones probablemente se debieran a ese factor. Por último se decidió dejar fuera de la investigación las traducciones de "The Cat the Walked by Himself" ya que, quizás por tratarse de un cuento perteneciente a la llamada literatura infantil, el análisis preliminar mostró que este texto presentaba menos problemas de comprensión que los otros 
dos.

De esta manera, el corpus quedó 55 traducciones elaboradas por 55 alumnos de Traducción Literaria 1. Una vez seleccionado el corpus, se dio paso al análisis exhaustivo y la recolección de datos de las producciones a través del método comparativo constante. Como primer paso, se leyeron todas las traducciones y se identificaron los pasajes de las mismas que mostraban dificultades de por parte de los estudiantes en la comprensión de la información del TF. Estos pasajes fueron codificados con una (C) al margen de las traducciones. Luego, se hizo una contabilización sencilla de los pasajes identificados y se seleccionaron aquellos que presentaban un desafío para un mayor número de estudiantes.

Nuestro análisis se centró en los problemas de comprensión del TF que intervienen en: a) el procesamiento de los niveles de representación de la información del texto fuente (estructura superficial, base del texto y modelo de situación); b) la interpretación del TF en tanto artefacto lingüístico, literario y soiciocultural. Por último, se analizó la relación que podía establecerse entre los problemas de comprensión y el desarrollo de la competencia traductora que participa de los procesos mencionados aportando las habilidades y conocimientos requeridos por la tarea. Por cuestiones de claridad expositiva, hemos hecho referencia a cada uno de los aspectos citados de manera separada si bien sabemos que, en la práctica, los procesos operan de manera concomitante para lograr una comprensión e interpretación adecuadas del texto fuente.

El análisis de las traducciones fue un proceso recurrente e iterativo que implicó la lectura y relectura de las traducciones completas y los pasajes seleccionados en reiteradas oportunidades en el transcurso de la investigación, la toma de notas, la transcripción de los pasajes seleccionados y de las reexpresiones que elaboraron los estudiantes para los pasajes seleccionados. Este proceso se repitió hasta lograr dar respuesta a los interrogantes que motivaron la investigación y hasta que la investigadora ya no pudo obtener nuevos datos y se consideró que se había alcanzado el nivel de saturación de la muestra.

A continuación, se presenta una sinopsis de la trama del cuento de Achebe utilizado en esta investigación y la consigna de trabajo a partir de la cual los estudiantes elaboraron sus producciones.

\subsubsection{Sinopsis de la trama del cuento "Dead Men's Path” de C. Achebe y consigna de trabajo}

“Dead Men’s Path" comienza con la designación del Sr. Obi, como nuevo director de una escuela en la aldea de Ani. Michael Obi y su esposa tienen grandes expectativas de que la experiencia sea un éxito y una oportunidad de dar a la escuela y a la comunidad nuevos aires de modernidad y de progreso. El flamante director insiste en la exigencia académica mientras que Nancy, su esposa, pone gran empeño en el cuidado de los jardines. Un día, mientras se encuentra admirando su obra, el Sr. Obi descubre que hay un viejo sendero que une el santuario y el cementerio de la comunidad local, el cual atraviesa el predio de la escuela. Horrorizado ante la posibilidad de que la inspección que espera se vea arruinada por alguna suerte de "juego tribal”, el Sr. Obi decide cerrar los accesos al sendero y se mantiene firme en su decisión a pesar de las explicaciones del sacerdote del pueblo en pos de que no introduzca 
ningún cambio. Según las creencias de la comunidad, el sendero es transitado por los bebés que llegan al mundo y por los muertos que visitan a los vivos. Días después de la clausura del sendero, una joven de la comunidad muere dando a luz. El sacerdote del pueblo ordena sacrificios para desagraviar a los ancestros. El Sr. Obi encuentra su escuela y sus jardines destruidos y la inspección resulta un fracaso cuando el supervisor registra una situación de guerra tribal como consecuencia del fervor desafortunado del nuevo director.

Resulta interesante notar la singularidad de la escritura de Achebe, considerado por muchos, como el padre de la literatura africana. Se trata de la obra de un poeta y novelista nigeriano aclamado internacionalmente por sus reflexiones sobre el impacto de la imposición de valores y costumbres occidentales sobre la sociedad africana tradicional. Achebe es, además, un autor de minorías, cuya lengua materna es el igbo pero que escribe su obra en inglés, la lengua oficial de Nigeria. A propósito de las relaciones entre el inglés y las lenguas nativas africanas, Achebe (1965) señala que el inglés permite comunicar el peso de la experiencia individual africana al mundo. En este sentido, la escritura de Achebe presenta un uso creativo de la lengua dominante (el inglés) que incorpora elementos y aspectos culturales vinculados a la lengua y cultura minoritaria (el igbo).

Según recuerda un artículo llamado "Chinua Achebe: A life in writing", publicado en The Guardian en el año 2010, en ocasión de la entrega del Man Booker International Prize, el jurado expresó que la obra de Chinua Achebe resignificaba los límites de la novela recreados por James Joyce para el siglo XX e iluminaba el camino a nuevos escritores que estuvieran en busca de nuevas palabras y nuevas formas.

Como parte de la cursada de Traducción Literaria 1, los estudiantes tuvieron que realizar la siguiente práctica de traducción:

Lea y traduzca el siguiente fragmento del cuento "Dead Men's Path" de Chinua Achebe, cuya traducción le ha sido encomendada por la cátedra libre de Estudios poscoloniales de la Universidad. Escriba su traducción en tinta dejando un espacio entre renglón y renglón. Por favor no escriba en los márgenes de la hoja.

Heavy sticks were planted closely across the path at the two places where it entered and left the school premises. These were further strengthened with barbed wire.

Three days later the village priest of Ani called on the headmaster. He was an old man and walked with a slight stoop. He carried a stout walking stick which he usually tapped on the floor, by way of emphasis, each time he made a new point in his argument.

"I have heard," he said after the usual exchange of cordialities, "that our ancestral footpath has recently been closed...”

"Yes," replied Mr. Obi. "We cannot allow people to make a highway of our school compound."

"Look, here, my son," said the priest bringing down his walking stick, "this path was here before you were born and before your father was born. The whole life of this village depends on it. Our dead relatives depart by it and our ancestors visit us by it. 
But most important, it is the path of children coming in to be born..." Mr. Obi listened with a satisfied smile on his face. "The whole purpose of our school," he said finally, "is to eradicate just such beliefs as that. Dead men do not require footpaths. The whole idea is just fantastic. Our duty is to teach your children to laugh at such ideas."

"What you say may be true," replied the priest, "but we follow the practices of our fathers. If you reopen the path we shall have nothing to quarrel about. What I always say is: let the hawk perch and let the eagle perch." $\mathrm{He}$ rose to go.

"I am sorry," said the young headmaster. "But the school compound cannot be a thoroughfare. It is against our regulations. I would suggest your constructing another path, skirting our premises. We can even get our boys to help in building it. I don't suppose the ancestors will find the little detour too burdensome."

"I have no more words to say," said the old priest, already outside.

\section{Resultados}

A continuación presentamos los resultados del análisis efectuado sobre 55 traducciones realizadas por estudiantes de Traducción Literaria 1 de una universidad nacional argentina como parte de las prácticas de traducción de la cursada. Las traducciones elaboradas por los estudiantes corresponden a un fragmento del cuento "Dead Men's Path" de Chinua Achebe (1930-2013). A partir del análisis del corpus, fue posible construir las categorías que se presentan a continuación. Tales categorías fueron elaboradas a partir de las recurrencias en las traducciones efectuadas por los estudiantes. Ese trabajo constituyó la base sobre la cual fue posible identificar los desafíos que presenta la lectura de textos literarios para su traducción a los estudiantes que constituyen la muestra de esta investigación. Hemos organizado nuestras observaciones en relación con las siguientes categorías: a) desafíos en relación con la elaboración de los distintos niveles de representación textual: superficie, base del texto y modelo de situación; b) desafíos en la interpretación del TF en sus dimensiones lingüística, literaria y sociocultural y c) desarrollo de la competencia traductora de los lectores-traductores en formación.

3.1 Desafios en relación con la elaboración de los distintos niveles de representación textual: superficie, base del texto y modelo de situación.

$\mathrm{Al}$ analizar las 55 traducciones realizadas por los estudiantes en respuesta a la tarea, se observa que los textos producidos logran reconstruir con relativo éxito algunos aspectos macroestructurales de la trama y del diálogo. En efecto, las traducciones propuestas por los estudiantes reflejan la clausura del sendero como el conflicto central del relato y dan cuenta de las posiciones antagónicas del sacerdote y del director de manera clara. Sin embargo, la comprensión del relato de Achebe presenta algunos desafíos para los lectores-traductores en 
formación que resulta de interés estudiar con cierto detalle.

$\mathrm{Al}$ comienzo del fragmento que constituye el TF seleccionado para la práctica de traducción, el texto alude a la clausura del camino que decide el Director de la escuela, el cual desencadenará el conflicto central del cuento, ya que esa decisión viola las normas y tradiciones de la comunidad igbo: "Heavy sticks were planted closelyacross the path at the two places where it entered and left the school premises. These were further strengthened with barbed wire". En este pasaje, 32 de las 55 traducciones de los estudiantes (58\%) evidencian dificultades precisamente en el punto en que el narrador destaca que el sendero atravesaba el predio de la escuela. En efecto, la interpretación de la cláusula "where it entered and left the school premises" registra las siguientes reexpresiones:

a) ... los dos lugares por los que se entraba y se salía del predio de la escuela

b) ... los dos lugares de entrada y salida del predio de la escuela

c) ... los dos lugares donde comenzaba y terminaba el predio de la escuela

En las reexpresiones citadas, se evidencia un establecimiento inadecuado de la referencia del pronombre "it" en el nivel de la superficie textual ${ }^{2}$. Cabe señalar que en este trabajo empleamos el adjetivo "adecuado" en el sentido corriente que adopta en la lengua española, en la que designa que algo o alguien es apropiado en un contexto determinado.

Respecto de la información que constituye la base del texto, las reexpresiones citadas no dan cuenta de algunos aspectos centrales de la narración. En efecto, en el cuento de Achebe, el sendero no lleva a la entrada y a la salida del predio de la escuela, sino a dos lugares sagrados de la comunidad local. Si bien esta información no forma parte del fragmento que los estudiantes deben traducir en el marco de esta práctica de traducción, es proporcionada al director por un docente de la escuela de manera explícita en otro pasaje del cuento, que los estudiantes han leído en su totalidad con anterioridad a la práctica de traducción. En consecuencia, una comprensión adecuada del cuento implica que esta información esté disponible para los lectores-traductores en formación como parte de la base del texto. Por otra parte, la centralidad de esta información en la trama del cuento implica una posición privilegiada en la estructura temática global por cuanto el bloqueo de la entrada y salida del sendero provoca el conflicto que constituye el nudo de la narración.

En el mismo pasaje, la comprensión de la palabra "premises" plantea sus propios desafíos en la elaboración de la estructura superficial del TF. El término "premises" tiene dos sentidos en inglés; esta palabra puede designar "a house or building, together with its land and outbuildings, occupied by a business or considered in an official context"(Oxford English Dictionary [OED], 2018) o el plural de "premise", esdecir, "a previous statement or proposition from which another is inferred or follows as a conclusion"(OED).En consecuencia, según el sentido que opere en el TF, la palabra "premises"podría, en funcióndel contexto de uso, traducirse al español como "predio" o como "premisas". Si bien en el cuento de Achebe,

\footnotetext{
${ }^{2}$ Cabe señalar que el adjetivo "adecuado" en esta tesis no se emplea en el sentido técnico de Toury ([1978]1995) quien define la noción de adecuación en relación con la decisión del traductor de ajustarse a las normas de la lengua y cultura fuente.
} 
"premises" refiere al predio de la escuela, en 25 de las 55 traducciones de "DMP" (45\%) "premises" se traduce como "premisas", lo cual da lugar a reexpresiones como "los lugares por donde entraba y salía de las premisas de la escuela" o "los lugares por donde entraba y salía de las premisas escolares".En relación con la base del texto, es interesante notar que la traducción de "premises" como "premisas" no permite derivar ninguna proposición que contribuya a la coherencia global de la base del texto.

Siguiendo con las dificultades que tienen los estudiantes en la elaboración de los distintos niveles de representación de la información textual en el cuento, la interpretación y traducción de los sustantivos comunes "children", "boys" y "priest" que representan algunos de los actores centrales del cuento aparece como otro aspecto problemático en nuestro corpus.

En un pasaje de la discusión que llevan adelante el director de la escuela y el sacerdote acerca de las creencias de la comunidad en medio del conflicto suscitado por la clausura del sendero ancestral, el Sr. Obi presenta el siguiente argumento: "Dead men do not require footpaths. The whole idea is just fantastic. Our duty is to teach your children to laugh at such ideas". Como es sabido, el sustantivo plural "children" admite diversos sentidos. Por un lado, en tanto forma plural del sustantivo "child", "children" evoca, en primer término, la noción de niños y niñas, sin distinguir el género. Por otro lado, el sustantivo plural se emplea habitualmente para referirse a los hijos varones y mujeres, nuevamente, sin que esto implique una distinción de género. Así, una interpretación apropiada del sustantivo "children” en un texto particular requiere de otros elementos de la superficie textual y de la base del texto que la sustenten. En este caso, en relación con las "pistas" que pueden obtenerse de la estructura superficial, el principal elemento que debe tenerse en cuenta es la presencia del adjetivo posesivo "your" en el sintagma nominal "yourchildren". En efecto, cuando la palabra "children" aparece precedida de un adjetivo posesivo en frases como "mychildren", "your children", "his/her children", "our children" o "their children", el adjetivo posesivo indica el sentido de la palabra como "hijos (varones y mujeres)". Sin embargo, 37 de las 55 traducciones del fragmento de "DMP" (67\%) optan por reformular "your children" como "sus niños" en la traducción del pasaje.

En el nivel de la base del texto, surgen nuevos elementos, ya que, en su intento por convencer al director para que libere el ingreso al sendero de los muertos de la comunidad, el sacerdote intenta explicarle al Sr. Obi la relevancia que ha tenido el sendero para la historia de la comunidad en un pasaje que abunda en referencias a la familia. En este contexto, cuando el director presenta el argumento de que esas ideas son absurdas, lo que intenta es, precisamente, cuestionar esa cadena generacional por la que los padres han comunicado estas creencias a sus hijos a través de la historia de la comunidad. En vista de estas referencias a la transmisión de ideas y valores culturales que se da de generación en generación, "sus hijos" contribuye a la coherencia global del pasaje. Por el contrario, "sus niños", no resulta adecuada para establecer ese marco de coherencia en el texto. Como hemos mostrado, la información presentada en los niveles de la estructura superficial y de la base del texto nos permiten establecer que "sus hijos" es una reformulación adecuada de la frase "yourchildren".

Al igual que "children", el sustantivo plural "boys" comprende distintos sentidos en inglés, tal como se pone en evidencia en el pasaje del cuento que citamos a continuación: 
"I am sorry," said the young headmaster. "But the school compound cannot be a thoroughfare. It is against our regulations. I would suggest your constructing another path, skirting our premises. We can even get our boys to help in building it. I don't suppose the ancestors will find the little detour too burdensome". (El subrayado es nuestro.)

En los principales diccionarios ingleses y estadounidenses (OED; Merriam-Webster.com Dictionary, s.f.), el término "boy" presenta, entre sus acepciones (además de la más difundida de niño varón), la posibilidad de utilizarlo para designar de manera peyorativa a los sirvientes negros. En el caso del cuento, la palabra "boys" parece hacer referencia a cierto personal con el que la escuela cuenta para tareas de mantenimiento. En español, por otra parte, y según el Diccionario de la Lengua Española(2017) (En adelante, DLE),"muchachos” puede designar a "un mozo que sirve de criado". Sin embargo, 43 de las55 traducciones efectuadas por los estudiantes (78\%) optan por las reexpresiones "jóvenes" o "niños", que resultan menos propicias, ya que ninguna de las dos indica la condición de trabajador del sujeto aludido ni, mucho menos, el tono peyorativo que puede estar implicado en "boys". Ahora bien, el texto de Achebe aporta pocos indicios en el nivel superficial y en el nivel de la base del texto. Después de todo, el director solo menciona a los muchachos cuando dice que ellos pueden ayudar a construir un sendero nuevo. Para llegar a una interpretación adecuada de "boys" es preciso tener conocimientos de este sentido del término: se trata de muchachos muy probablemente nativos que trabajan de manera informal para la escuela; estos conocimientos se encuentran, fundamentalmente, en el nivel del modelo de situación.

Por otra parte, 27 traducciones del fragmento de "DMP" (49\%) presentan dificultades a la hora de interpretar y traducir la palabra "priest" en el cuento de Chinua Achebe, que se traduce de manera errónea como "cura". En su definición de la palabra "priest", el OED arroja los siguientes sentidos que resultan relevantes para esta discusión: "An ordained minister of the Catholic, Orthodox, or Anglican Church, authorized to perform certain rites and administer certain sacraments" y, como un sentido asociado al primero, "a person who performs religious ceremonies and duties in a non-Christian religion". El DEL por su parte, define "sacerdote" de las siguientes maneras: "En la Iglesia Católica, hombre ordenado para celebrar el sacrificio de la misa y realizar otras tareas propias del ministerio pastoral", y "persona dedicada y consagrada a hacer, celebrar y ofrecer sacrificios". Como señala la entrada del DLE, en español este término designa, de manera exclusiva, al sacerdote católico y, por ello, resulta una traducción inadecuada de "priest" a la luz del modelo de situación que permite elaborar el cuento de Achebe. Para construir un modelo de situación adecuado, el lector-traductor debe poner en juego conocimientos sobre el contexto sociocultural del cuento, más específicamente en relación con los sistemas religiosos de las comunidades indígenas nigerianas.

En nuestro corpus, el sintagma nominal "dead men" es reformulado en 15 traducciones (27\%) como "hombres fallecidos", lo cual da lugar a reexpresiones como: "Los hombres fallecidos no necesitan senderos". El caso de "dead men" resulta particularmente interesante, ya que es parte del título del cuento, lo cual le brinda, siguiendo el modelo de van Dijk y Kintsch, relevancia macroestructural. El DLE define "fallecido" como un adjetivo en desuso que significa 
"desfallecido, debilitado". Por otra parte, "muerto" registra como primera acepción el sentido de "que está sin vida". Podemos decir, entonces, que la elección de "muertos" es más específica en relación con el contenido proposicional de la base del texto y que la elección de "fallecidos" en menos adecuada en el contexto del cuento de Achebe.

3.2 Desafíos en la interpretación del TF en sus dimensiones lingüistica, literaria y sociocultural.

Las categorías de artefacto lingüistico, artefacto literario y artefacto sociocultural de Holmes ([1978]1988) nos aportan las herramientas para realizar nuevos análisis de las dificultades de comprensión del TF que se ponen en evidencia en las traducciones de los estudiantes. Recordemos que, según el autor, la lectura del TF, en tanto artefacto lingüístico, nos permite acceder a su información contextual, mientras que, como artefacto literario y sociocultural, el TF reúne información intertextual y situacional, respectivamente.

Aplicando las categorías de Holmes, los problemas que hemos identificado en torno a la interpretación y reexpresión del pronombre "it", el sustantivo común "premises", y la frase nominal "your children" representan dificultades en la interpretación del TF en su dimensión lingüística. Los desafíos que presenta la presencia de "boys" y "priest” en el TF, por otra parte tienen, además de una dimensión lingüística, una dimensión sociocultural que revela una interpretación inadecuada de la información situacional del TF.Por último, las traducciones que presentan "hombres fallecidos" como reexpresión de "dead men" resultan inadecuadas en función de la información intertextual que aporta el TF en tanto artefacto lingüístico y literario.

\subsection{Desarrollo de la competencia traductora de los lectores-traductores en formación.}

Todos los problemas de comprensión analizados hasta aquí se relacionan, por un lado,con el desarrollo de la subcompetencia bilingüe de los traductores en formación, quienes no logran identificar el significado o la referencia correcta de los elementos de la estructura superficial del TF. Por el otro, observamos que los estudiantes tampoco logran identificar ni solucionar losproblemas a través de la subcompetencia estratégica. Una solución adecuada para tales dificultades de comprensión requeriría, además, de la subcompetencia instrumental en el uso eficaz de libros de referencia (diccionarios monolingües y bilingües), particularmente en los casos de los sustantivos comunes"premises", "boys" y "priest", y del adjetivo "dead".

Ahora bien, las elecciones inadecuadas de los estudiantes, especialmente en torno a la interpretación y traducción de "priest", "boys" y "deadmen" en el pasaje de "DMP” muestran, asimismo, problemas en relación con la subcompetencia extralingüística, que aporta los conocimientos socioculturales y literarios requeridos en la lectura de textos literarios. Estos conocimientos declarativos resultan necesarios para construir modelos de situación adecuados que den cuenta en la traducción de la información del TF como artefacto literario y sociocultural.

Hasta aquí, hemos descripto los resultados de nuestra investigación en relación con las traducciones efectuadas por los estudiantes del fragmento del cuento "DMP”. A continuación, presentamos nuestras observaciones sobre las 55 traducciones del fragmento del cuento "TFS". 


\section{Discusión y conclusiones}

La investigación que presentamos en este trabajo indaga los desafíos que presenta la lectura de textos literarios para su traducción en la formación de traductores públicos. Para ello, hemos analizado un corpus de 55 traducciones realizadas por estudiantes de Traducción Literaria 1 de la carrera de Traductorado Público en Lengua Inglesa de una universidad nacional argentina. A través de un estudio no experimental, transversal y exploratorio basado en traducciones elaboradas por los estudiantes de un fragmento del cuento "Dead Men's Path de C. Achebe, hemos identificado un conjunto de desafíos que, según entendemos, ponen de manifiesto la importancia de la lectura en el éxito del proceso de traducción (Averbach, 2011; Dancette (1995) y Kussmaul (1996, 1997).

En el marco de este trabajo, el término "desafío" hace referencia a los retos, obstáculos o escollos que presenta la lectura a los estudiantes de traducción literaria; por otra parte, la lectura comprende el análisis del TF que constituye la primera etapa del proceso traductor (VázquezAyora, 1977; Nida, 1969; Krings, 1986ª Jääskeläinen, 1999; Norberg, 2003; Jakobsen, 2003); y la traducción literaria es entendida como la traducción de originales "literarios" que intenta preservar o recrear las intenciones o efectos estéticos del original (Delabatista, 2011).

En consideración de los resultados obtenidos, los desafíos que enfrentan los traductores en formación serían los que se detalla a continuación.

4.1 Desafios en relación con la elaboración de los distintos niveles de representación de la información textual (estructura superficial, base del texto y modelo de situación)

Con respecto a la estructura superficial, los problemas analizados en relación con la interpretación y reexpresión de elementos del TF como el pronombre "it" y "your children" en el cuento de Achebe indican la dificultad que enfrentan los estudiantes para utilizar la información sintáctica del TF como una herramienta para identificar el sentido adecuado que adquieren las palabras del TF en su contexto particular.

De nuestro análisis puede desprenderse, asimismo, la preponderancia de un desafío que juega un papel importante en los problemas de comprensión que enfrentan los estudiantes de traducción que participaron de nuestra investigación. Este desafío está relacionado con el hecho de que en el lenguaje una palabra puede tener varios significados. Este fenómeno, que se conoce como polisemia, aparece como un problema frecuente en nuestro corpus. La interpretación y reexpresión de ejemplos como "children" y "boys" ponen de manifiesto, a nuestro entender, la ubicuidad de la polisemia en el lenguaje y los retos que la misma presenta al lector-traductor en formación.

Otro fenómeno que aparece como problemático en nuestro corpus se da en el espacio de relación entre la lengua fuente (LF) y la lengua meta (LM). En efecto, la interpretación de términos que resultan morfológicamente similares pero semánticamente diferentes entre las lenguas presenta desafíos a los traductores en formación. Estas palabras, que comúnmente se conocen como falsos cognados se ven representadas en nuestro corpus por el término "premises". 
La interpretación de los falsos cognados implica desestimar la semejanza morfológica entre dos términos de la LF y la LM y dar prioridad a su contenido semántico para identificar el sentido adecuado que opera en el texto. El ejemplo de "premises" en nuestro análisis del cuento de Chinua Achebe evidencia las dificultades que pueden enfrentar los estudiantes en relación con la interpretación y la traducción de estas palabras en los procesos de selección léxica. En el caso particular de "premises" en nuestro corpus, las dificultades se incrementan al tratarse de un término polisémico que es, además, un falso cognado. En estos casos, se observa que los estudiantes no tienen aún desarrollada la capacidad de identificar e interpretar información relevante del TF. Esta capacidad es señalada por Shreve y Diamond (1997) y Bell (1998) como una característica de las habilidades de lectura de los traductores profesionales.

Al momento de elegir la palabra que se utilizará en la reexpresión en el TM, los estudiantes favorecen los sentidos más frecuentes de los términos en el TF, aquellos que, en consecuencia, los diccionarios ubican en las primeras posiciones de sus definiciones. En nuestro corpus, este es el caso en la interpretación y traducción de "boys", "premise", "children" y "priest".

Los desafíos relacionados con la polisemia del lenguaje, los falsos cognados y, de manera más general, la selección léxica que se da en los procesos de traducción pone de manifiesto la profundidad de la cuestión del significado en los procesos de traducción. La ubicuidad del dialogismo y la heteroglosia que Bajtín reconoce como propiedades de todo discurso, resultan particularmente desafiantes en el discurso literario, ya que la pluralidad de significados es constitutiva de este discurso (Anchía, 2006). Por otra parte, si bien las observaciones que hemos hecho hasta aquí son válidas para la lectura de textos literarios en general, estos procesos complejos de (re)construcción parecieran ponerse de manifiesto de maneras particularmente sensibles en la traducción en la que el lector-traductor debe saldar la búsqueda de significado en la elección de una palabra para el texto meta.

Los resultados obtenidos ponen de manifiesto la importancia de la superficie textual en la lectura para la traducción literaria. En este sentido, nuestros resultados están en línea con la observación de Shreve y Lacruz (2017), señalan que la representación palabra por palabra (verbatim) del TF ejerce una influencia mayor y más duradera de lo que los modelos de comprensión suelen reconocer.

En relación con los desafíos que presenta la elaboración de la base del texto, el análisis de nuestro corpus indica que los estudiantes tienen dificultades para integrar la información proposicional del TF en la construcción de una macroestructura coherente. Más específicamente, los problemas que se presentan en relación con la interpretación y reexpresión de "premises" parecieran mostrar que los estudiantes tienen problemas para iluminar las elecciones locales con información extraída de otros pasajes del texto. Estos problemas en el nivel de la base del texto se trasladan a la construcción de la macroestructura, ya que las dificultades para establecer un contenido proposicional adecuado afectan las posibilidades de elaborar una estructura proposicional organizada jerárquicamente que dé cuenta del contenido temático global del texto. En este sentido, es posible pensar que los estudiantes no han desarrollado aún la capacidad que de interpretar las pistas comunicativas del TF para componer una interpretación global coherente del mismo que es característica de los traductores expertos 
(Alves y Gonçalves, 2007).

El tercer nivel de la representación de un texto, que constituye el modelo de situación, combina la información del texto con los conocimientos del lector y cumple un rol fundamental en el procesamiento del discurso y la adquisición de conocimiento acerca del mundo (van Dijk, 1978; 1980; 2014; van Dijk y Kintsch 1983; Kintsch, 1988 y 1998).

En nuestra investigación encontramos varios ejemplos de la influencia del modelo de situación en la construcción de la comprensión del TF y los desafíos que enfrentan los estudiantes en la construcción de modelos de situación en relación con textos culturalmente ajenos. Así, por ejemplo, las razones que orientan la interpretación de "boys" en relación con personal -probablemente informal- de procedencia local con el que cuenta la escuela para tareas de mantenimiento se encuentran en el modelo de situación. En este sentido, podemos decir que las pistas que permiten establecer "muchachos" como una reformulación más adecuada en español remiten a información situacional del texto fuente. Son los conocimientos de la historia de Nigeria, del dominio británico, de las relaciones entre los nativos y los colonizadores británicos en ese contexto, los que les permiten a los lectores-traductores construir un modelo de situación adecuado para el cuento. Este modelo, a su vez, contribuye a una interpretación precisa y completa de la palabra "boys", que es posible gracias a un procesamiento top-down de la información del texto.

Algo similar se observa en relación con la interpretación y traducción de "priest". El desafío principal que implican la interpretación y la traducción de "priest" se relacionan con dificultades que afectan el nivel del modelo de situación. Para construir un modelo de situación adecuado, el lector-traductor debe poner en juego conocimientos sobre el contexto sociocultural del cuento de Achebe, más específicamente en relación con los sistemas religiosos de las comunidades indígenas nigerianas. Creemos que la falta de este tipo de marcos, que Holmes ubica dentro de la información situacional que aporta el texto como artefacto sociocultural, determina en gran medida la elección de "cura" en las traducciones de los estudiantes.

\subsection{Desafios en la interpretación del TF como artefacto lingüístico, literario y sociocultural.}

En relación con los desafíos que el TF plantea a los estudiantes en tanto artefacto lingüístico, sociocultural y literario, es posible retomar algunas observaciones realizadas más arriba. En efecto, las dificultades que imponen fenómenos como la polisemia y los falsos cognados que fueron analizadas en el apartado anterior, pueden interpretarse en relación con la elaboración de la información contextual del TF. Podemos pensar que los ejemplos analizados arriba en atención a la interpretación y reexpresión de "boys", y "children" en el cuento de Achebe a nivel de la estructura superficial, se relacionan con la interpretación del TF en tanto artefacto lingüístico.

El corpus de análisis presenta, asimismo, un buen ejemplo de los desafíos que ofrece el TF en tanto artefacto literario que forma parte, a su vez, de un continuo literario. El sintagma "dead men" que integra el título del cuento de Achebe, tiende lazos de intertextualidad con grandes obras de la literatura como, por ejemplo, el cuento "The Dead" de James Joyce, y eventualmente con el cuento "El muerto" de Jorge Luis Borges y "El hombre muerto" de 
Horacio Quiroga. La intertextualidad que vehiculiza la frase "los muertos", sin embargo, no se limita a la literatura ya que también evoca, potencialmente, El libro de los muertos, una colección de sortilegios que los antiguos egipcios depositaban en las tumbas para ayudar a los muertos a sobrellevar el Juicio de Osiris. Las traducciones que presentan "hombres fallecidos" como reexpresión de "dead men" no dan cuenta de este continuo literario del que el cuento de Achebe forma parte. El desafío consiste precisamente en interpretar la información intertextual del TF que permite hacer estas conexiones, las cuales requieren conocimientos amplios acerca de los géneros y tradiciones literarias que, de algún modo, entran en el gran diálogo que representa el desarrollo de la literatura.

4.3 Por otra parte, la interpretación del TF requiere que se decodifique y se resignifique la información situacional del TF.

Esta información, que representa las huellas que la pertenencia a un tiempo y espacio históricos dejan en el TF es indispensable a la hora de interpretar algunos de los pasajes de los cuentos. Los ejemplos que citamos más arriba en relación con la interpretación de "boys" y "priest" resultan buenos ejemplos de los retos que implica la comprensión de estos aspectos socioculturales del TF. A la luz de estos ejemplos, puede concluirse que las dificultades que presentan los estudiantes están asociadas, al menos en parte, a conocimientos insuficientes de los contextos socioculturales que se ven representados en los textos. ¿Qué pueden decirnos los desafíos identificados hasta aquí acerca del desarrollo de la competencia traductora de los estudiantes, lectores-traductores en formación?

El corpus de esta investigación presenta problemas de comprensión del TF en los que intervenienen prácticamente todas las subcompetencias que conforman la competencia traductora. Más específicamente, los problemas de corte lingüístico que analizamos en atención a la estructura superficial y a la información contextual del TF en tanto artefacto lingüístico indican que la subcompetencia bilingüe de los estudiantes requiere mayor desarrollo para hacer frente a los desafíos que representa la interpretación de los textos del corpus.

El análisis de los problemas que tienen los estudiantes para interpretar los rasgos lingüísticos de expresiones como "boys", "premises" o "children” en el texto nigeriano podrían mostrar que los estudiantes no cuentan aún con un conocimiento preciso y completo de las palabras del TF que les permita reexpresarlas de manera adecuada en el texto meta. El análisis del corpus, por otra parte, nos permite cuestionar si la subcompetencia bilingüe que se pone en juego en la traducción es equiparable, como la describe Hurtado Albir, a las competencias en el uso de distintas lenguas que despliega un sujeto no-traductor. Desde la perspectiva de PACTE, la subcompetencia bilingüe es considerada entre las subcompetencias no exclusivas del traductor. Nuestros ejemplos, sin embargo, nos permiten pensar que la traducción, especialmente la traducción literaria, posiblemente requiere de un tipo de subcompetencia bilingüe que no es equiparable, al menos en su totalidad, a la competencia de usuarios de las lenguas que no son traductores. Si bien el grupo PACTE establece una clara diferenciación entre la competencia bilingüe de un sujeto no traductor y los conocimientos y habilidades de un traductor, que integra en el concepto de competencia traductora, la subcompetencia bilingüe 
es considerada, desde la perspectiva del Grupo, entre las subcompetencias no exclusivas del traductor, junto con la subcompetencia extralingüística y los componentes psicofisiológicos (memoria, atención, entre otros). En este sentido y como se recordará, Hurtado Albir (1996, 2001) define la competencia bilingüe como los conocimientos (básicamente operativos) pragmáticos, psicolingüísticos, textuales y del código lingüístico necesarios para la comunicación en dos lenguas.

Las dificultades que tienen los estudiantes, hablantes con un nivel avanzado de inglés como lengua extranjera, en la interpretación de palabra de altísima frecuencia nos hacen pensar que la traducción probablemente requiere de un tipo de "bilingüismo" al menos sutilmente diferente al que se establece en otras situaciones comunicativas. En este sentido, puede resultar apropiado hablar de una subcompetencia bilingüe del traductor que reúna los conocimientos sobre las lenguas fuente y meta que resultan necesarios, no solamente para la comunicación entre lenguas, sino, de manera específica, para la traducción entre lengua y culturas. La caracterización de este tipo específico de subcompetencia podría ser el objetivo de investigaciones futuras.

En relación con la subcompetencia instrumental, el corpus parece indicar que los estudiantes no logran hacer un uso eficaz de las herramientas a su alcance (diccionarios, enciclopedias, entre otros). Como señalamos en el capítulo 4 que reúne los resultados de la investigación, algunos de los problemas que se suscitan en la interpretación de los TF podrían haber sido resueltos mediante el uso efectivo de los diccionarios bilingüe y monolingüe que están a disposición de los estudiantes en estas tareas de práctica de la traducción. Este es el caso de la interpretación de elementos como "boys", y "priest", por citar solo algunos ejemplos. En estos casos, los estudiantes parecen favorecer los primeros sentidos que registran las palabras en los diccionarios en detrimento de sentidos que, por ser menos habituales, se registran más abajo en las entradas de los diccionarios. Esta cuestión, que no puede ser puesta a prueba en el marco de esta investigación, puede ser abordada en investigaciones futuras.

El desarrollo de la subcompetencia estratégica es clave en la formación de traductores ya que, como señalan Hurtado Albir y el grupo PACTE, la misma regula el proceso traductor, identifica los problemas y procura encontrar soluciones. En consecuencia, cuando se dan errores en la traducción, puede pensarse que la subcompetencia estratégica ha fallado de algún u otro modo. Lo que pone de manifiesto el análisis de nuestro corpus, es que los estudiantes no logran identificar ciertos signos que evidencian problemas de comprensión en sus traducciones. Por ejemplo, parecieran no advertir las "incongruencias" que aparecen en sus textos meta como resultado de selecciones léxicas poco adecuadas. La identificación de estas "malas elecciones", que se relacionan con las dificultades para interpretar y reexpresar el TF que tratamos en esta investigación, podrían ser el punto de partida para revisar el proceso e intentar encontrar una mejor solución. Excede al marco de esta investigación poder establecer en qué punto del proceso y por qué mecanismos precisos falla la subcompetencia estratégica; pero estamos en condiciones de afirmar que en los casos analizados, se observan problemas. En el futuro pueden diseñarse trabajos para indagar más profundamente la incidencia de la subcompetencia estratégica en los problemas de comprensión del TF en la traducción literaria.

La subcompetencia extralingüística entra en juego, de manera crucial, en relación con 
los conocimientos literarios y socioculturales que requiere el TF para su comprensión. En efecto, la construcción de un modelo de situación adecuado, indispensable en el proceso traductor, requiere de una variedad de conocimientos y marcos que Holmes denomina información situacional - cuando se trata de conocimientos socioculturales - e información intertextual cuando se trata de conocimientos literarios. Las traducciones que hemos analizado en este trabajoindican que la subcompetencia extralingüística de los estudiantes requiere mayor desarrollo para poder hacer frente a los desafíos que presenta la lectura de textos literarios para su traducción. En el Plan de Estudios de los estudiantes que elaboraron las traducciones de nuestro corpus, este tipo de pertenece al ámbito de las materias de literatura e historia que deben cursar los estudiantes de manera obligatoria y optativa. Sin embargo, pensamos que este tipo de subcompetencia requiere, además, algún tipo de formación, enseñanza o entrenamiento específico que explore la aplicación de estos conocimientos a la tarea traductológica.

A lo largo de esta investigación hemos identificado los desafíos que enfrentan los estudiantes de Traducción Literaria 1 en la comprensión de textos literarios para su traducción. Es posible que nuestros resultados muestren que los traductores en formación no dedican suficiente atención a la lectura (Englund Dimitrova, 2005). Por otra parte, nuestra investigación pone en evidencia el carácter exhaustivo de la lectura en la traducción literaria que, en línea con Díaz-Diocartz (1985), implica explorar el TF de un modo mucho más pormenorizado que la lectura que realiza el crítico o el lector ordinario.

Nuestro estudio transversal, cualitativo, de carácter exploratorio, no permite proyectar de manera directa los resultados obtenidos a otras realidades. No obstante, la observación y el análisis del corpus nos ha permitido cumplir con el objetivo de identificar los desafíos que enfrentan los estudiantes de la muestra en la primera etapa del proceso de traducción. El repertorio de problemas y retos que hemos encontrado puede servir de puntapié inicial para nuevas investigaciones en otros contextos.

En el desarrollo de nuestro trabajo hemos buscado caracterizar los desafíos de comprensión que se reflejan en nuestro corpus. Los resultados que hemos obtenido no nos permiten señalar problemas que sean exclusivos de los traductores en formación, sino que puede tratarse de desafíos que alcancen a otros lectores en la comprensión de textos en lenguas extranjeras o segundas. Sin embargo, según creemos, los problemas de comprensión adquieren una relevancia singular en la traducción ya que suelen resultar en decisiones poco acertadas en la producción del texto meta. La interpretación del estudiante de traducción se materializa en una textualidad diferente y la construcción del TM requiere que el estudiante dé cuenta de todos los elementos que componen el TF en un proceso que es exhaustivo. En su traducción, se evidencia la lectura efectuada, que debe dar cuenta de todos y cada uno de los aspectos del texto.

Por último, el hecho de no haber podido realizar una triangulación de la información que nos permita contar con otras fuentes de información sobre los problemas estudiados es una limitación de esta investigación. En efecto, hubiera resultado útil tener la posibilidad de entrevistar a los estudiantes a través de grupos focales para poder indagar sobre sus percepciones, creencias y experiencias de los temas tratados en esta investigación. En este sentido, se espera que futuras investigaciones puedan profundizar en la comprensión de los 
desafíos que presenta la traducción literaria a los traductores en formación.

\section{Referencias}

ACHEBE, C. Dead Men's Path. In: Girls at War and Other Stories. Reimpreso y usado con el permiso de Harold Ober Associates Incorporated, 1972-1973 [1953].

ACHEBE, C. The African Writer and the English Language. In: Morning Yet on Creation Day. Londres: Heinemann, 1965.

ALVES, F.; GONÇALVES, J. Investigating the conceptual-procedural distinction in the translation process: A relevance-theoretic analysis of micro and macro translation units. In: EHRENSBERGER, M. et al. (eds.). Interdisciplinarity in Translation and Interpreting Process Research. Ámsterdam /Filadelfia, PA: Benjamins, 2015.

ANGELOU, M. I Know Why the Caged Bird Sings. Nueva York: Random House, 1969.

ATTRIGE, D. The singularity of literature. Londres: Routledge, 2004.

AVERBACH, M. Traducir literatura: una escritura controlada. Córdoba: Comunicarte, 2011.

AYUPOVA, R. Pretranslation Text Analysis as a Part of Translation Process. Social and Behavioral Sciences, 3(1), pp. 213-216, 2013. Recuperado de: <https://www.sciencedirect.com/science/article/pii/S1877042814037975?via\%3Dihub>

BAJTÍN, M. Estética de la creación verbal. México D.F.: Siglo XXI Editores, 1982 [1952-3].

BARNETT, M. More than meets the eye. NJ: Prentice Hall, 1989.

BASSNETT, S. Translation Studies (ed. rev.). Londres y Nueva York: Routledge, 2002 [1980].

BASSNETT, S.; LEFEVERE, A. (eds.). Translation, History and Culture. Londres y Nueva York: Routledge, 1990.

BECKETT, S. Waiting for Godot. Nueva York: Grove Press, 1953.

BELL, R. Psycholinguistic/cognitive approaches. In: BAKER, M. (ed.). Routledge, 1998.

ENCYCLOPEDIA of Translation Studies. Londres/Nueva York: Routledge.

BELOVA, Y. Interpretation Strategies as Constituents of Pre-Translation Analysis. 2015. Recuperado de: <https://cyberleninka.ru/article/v/interpretation-strategies-as-constituents-of-pretranslation-analysis>

BORGES, J.L. El muerto. In: El Aleph. Buenos Aires: Emecé, 1957.

BERNHART, E.B. Reading development in a second language: Theoretical, empirical and classroom perspectives. Norwood NJ: Ablex, 1991.

BOASE-BEIER, J. A Critical Introduction to Translation Studies. Londres/Nueva York: Continuum, 2011.

BOASE-BEIER, J. Knowing and not knowing: Style, intention and the translation of a Holocaust Poem, In: Comparative Critical Studies 2(1), pp. 96-104, 2004.

CARLINO, P. Escribir, leer y aprender en la universidad: Una introducción a la alfabetización académica. Buenos Aires: Fondo de Cultura Económica, 2005. 
CARRELL, P. Interactive text processing: Implications for ESL/second language reading classrooms. In: CARRELL, P.; DEVINE, J.; ESKEY, D. (eds.). Interactive Approaches to Second Language Reading. Cambridge: CUP, 1988.

CHOMSKY, N. Aspects of a Theory of Syntax. Cambridge, Massachusetts: MIT Press, 1965.

COLE, G.D.H. British Working Class Politics. Londres: Routledge \& Sons, 1941.

DELABASTITA, D. Literary translation. In: GAMBIER, Y.; VAN DOORSLAER, L. (eds.). Handbook of translation studies. Amsterdam: John Benjamins, 2011.

DELISLE, J. Iniciación a la traducción: Enfoque interpretativo. Teoría y Práctica. Caracas: UCV, 1997 [1985].

DÍAZ-DIOCARTZ, M. Translating Poetic Discourse: Questions on Feminist Strategies in Adrienne Rich. Ámsterdam/Filadelfia: John Benjamins, 1985.

DICIONARIO de la lengua española. Real Academia Española. Recuperado de: www.rae.es.

DROOP, M.; VERHOEVEN, L. Background knowledge, linguistic complexity, and second language reading comprehension. Journal of Literacy Research, 30, pp. 253-271, 1998.

ENGLUND DIMITROVA, B. Expertise and Explicitation in the Translation Process. Ámsterdam/Filadelfia: Benjamins, 2005.

FLURKEY, A.; XU, J., (eds.). On the revolution of reading: The selected writings of Kenneth S. Goodman. Portsmouth: Heinemann, 2003.

HALLIDAY, M. A. K. Language as a Social Semiotic. Londres y Nueva York: Arnold, 1978.

HATIM, B. Teaching and Researching Translation. Londres y Nueva York: Routledge, 2013 [2011].

HATIM, B.; MASON, I. Discourse and the Translator. Londres y Nueva York: Longman, 1990.

HERNÁNDEZ SAMPIERI, R.; FERNÁNDEZ COLLADO, C.; BAPTISTA LUCIO, P. Metodología de la investigación. México D.F.: McGraw-Hill, 2014.

HOLMES, S. The Name and Nature of Translation Studies. In: Translated Papers on Literary Translation and Translation Studies. Introducción de R. van de Broeck. Ámsterdam: Rodopi, 1988 [1972].

HOLMES, S. Translated Papers on Literary Translation and Translation Studies. Ámsterdam: Rodopi. Introducción de R. van den Broeck, 1988.

HOLMES, S. Describing Literary Translations. In: Translated Papers on Literary Translation and Translation Studies, Ámsterdam: Rodopi. Introducción de R. van de Broeck, 1988 [1978].

HURTADO ALBIR, A. (ed.). La enseñanza de la traducción. Castelló: Universitat Jaume, 1996.

HURTADO ALBIR, A. Traducción y traductología: Introducción a la traductología. Madrid: Cátedra, 2001.

JÄÄSKELÄINEN, R. Tapping the process: An Explorative Study of the Cognitive and Affective Factors Involved in Translating. Joensuu: Joensuun Yliopisto, 1999.

JAKOBSEN, A. L. Translation Process Research. In: SCHWIETER, J.A.; FERREIRA, A. (eds.) The Handbook of Translation and Cognition. Hoboken: Wiley Blackwell, 2017.

JAKOBSEN, A. L. Effects of think aloud on translation speed, revision, and segmentation. In: ALVES, F. (ed.), Triangulating Translation. Ámsterdam /Filadelfia, PA: Benjamins, 2003. 
JAKOBSEN, A. L.; JENSEN, K. T. H. Eye movement behaviour across four different types of reading task. In: GÖPFERICH, S.; JAKOBSEN, A. L.; MEES, I. M. (eds.), Looking at Eyes: EyeTracking Studies of Reading and Translation Processing. Copenhagen Studies in Language (Book 36). Copenhagen: Samfundslitteratur, 2008.

JOYCE, J. The Dead. En Dubliners. Clayton, Delaware: Prestwick House, 2006 [1914].

KINTSCH, W. The use of knowledge in discourse processing: a construction-integration model. In: Psychological Review, 95, pp. 163-182, 1988.

KINTSCH, W. Comprehension: A Paradigm for Cognition. Cambridge. CUP, 1998.

KIPLING, R. The cat that walked by himself. In: Just So Stories. Londres: Wordsworth Editions, 1993 [1902].

KRINGS, H. P. Was in denKöpfen von Übersetzern vorgeht. Tübingen: Narr, 1986.

KRINGS, H.P. The use of introspective data in translation. In: FAERCH y CASPER (eds.) Introspection in Second Language Research. Clevedon: Multilingual Matters, 1987.

LÖRSCHER, W. Process Oriented Research into Translation and Implications for Translation Teaching. In: TTR: Traduction, Terminologie, Redaction 5(1), pp. 145-161, 1992.

MALIK, A. A. A psycholinguistic analysis of the reading behavior of EFL-proficient readers using culturally familiar and culturally nonfamiliar expository texts. In: American Educational Research Journal, 27, pp. 205-223, 1995.

MARTÍN DE LEÓN, C. Translation in the wild: Traductología y cognición situada. In: PEGENAUTE, L.; DECESARIS, J.; TRICÁS, M.; BERNAL, E. (eds.). La traducción del futuro: mediación lingüística y cultural en el siglo XXI. Vol. II. La traducción y su entorno. Barcelona: PPU, 2008.

MERRIAM-WEBSTER Online Dictionary. Recuperado de: <https://www.merriamwebster.com/ >

MUNDAY, J. Introducing Translation Studies. Londres y Nueva York: Routledge, 2001.

NIDA, E.A. Toward a Science of Translating. Leiden: E. J. Brill, 1964.

NIDA, E.A.; TABER, C.R. The Theory and Practice of Translation. Leiden: E. J. Brill, 1969.

NIDA, E.A. Principles of Correspondence. In: VENUTI, L. (ed.) The Translation Studies Reader. 2. ${ }^{\text {a }}$ edición. Nueva York y Londres: Routledge, 2006 [2000].

NORBERG, U. Übersetzen mit doppeltem Skopos. Eine empirische Prozess- andProduktstudie. =Studia Germanica Upsaliensia42. Uppsala: Uppsala University, 2003.

NORD, C. Text Analysis in Translation. Theory, Method and Didactic Application of a Model for Translation Oriented Text Analysis. Translated from the German by Christiane Nord and Penelope Sparrow. Ámsterdam/Atlanta GA: Rodopi, 1991.

OXFORD English Dictionary. Recuperado de: <https://en.oxforddictionaries.com/>

PACTE. Building a Translation Competence Model. In: ALVES, F. (ed.).Triangulating Translations in Process Oriented Research. Ámsterdam: John Benjamins, 2003.

PHILLIPS, J.K. Practical implications of recent research in reading. Foreign Language Annals, 17, pp. 285-296, 1984. 
PILKINGTON, A. Poetic effects: A relevance Theory Perspective. Ámsterdam/Filadelfia: Benjamins, 2000.

PORTO, M. Un estudio exploratorio de la comprensión cultural en la lectura en inglés como lengua extranjera (ILE) en Argentina. (Tesis de doctorado, en línea). Universidad Nacional de La Plata. Facultad de Humanidades y Ciencias de la Educación, 2011. In: Memoria Académica. Recuperado de: 〈http://www.memoria.fahce.unlp.edu.ar/tesis/te.425_es/te.425_es.pdf.>

QUIROGA, F. El hombre muerto. In: A la deriva y otros cuentos. Montevideo: CEAL, 1968.

REISS, K.; VERMEER, H. J. Grundlegung einer allgemeinen Translationstheorie. Tübingen: Niemeyer, 1984.

RISKU, H.; WINDHAGER, F. Extended translation. A sociocognitive research agenda. Target, 25(1), 33-45, 2013. Repr. In: EHRENSBERGER et al. (eds.), Interdisciplinarity in Translation and Interpreting Process Research. Amsterdam, Filadelfia, PA: Benjamins, 2015.

ROSS, H. Holograming in a Robert Frost Poem: The still point. In: The Linguistic Society of Korea (eds.). Linguistics in the morning calm. Seúl: Hanshin Publishing, 1982.

RUMELHART, D.E. Schemata: The Building Blocks of Cognition. In: SPIRO, R.J.; BRUCE, B.C.; BREWER, W.F. (eds.). Theoretical issues in reading comprehension: perspectives from cognitive psychology, linguistics, artificial intelligence, and education. Hillsdale NJ: Lawrence Erlbaum, 1980.

SANTOS, A. C. El texto literario: aportaciones a la enseñanza del español como lengua extranjera. In: Actas del VI Seminario de dificultades específicas para la enseñanza del español a lusohablantes: el texto literario en la enseñanza del español como lengua extranjera. Brasilia, 1998.

SELESKOVITCH, D. Interpreting for International Conferences Traducción y adaptación al inglés de Seleskovitch (1968), París. Washington D.C.: Pen and Booth, 1978.

SHREVE, G. M.; LACRUZ, I. Aspects of a cognitive model of translation. In: SCHWIETER, J.W.; FERREIRA, A. (eds.). The Handbook of Translation and Cognition. Hoboken: Wiley Blackwell, 2017.

SHREVE, G. M.; SCHÄFFNER, C.; DANKS, J. H.; GRIFFIN, J. Is There a Special Kind of "Reading" for Translation? An Empirical Investigation of Reading in the Translation Process. Target, 5 (1), pp. 21-41, 1993.

SHREVE, G.; DIAMOND, B. J. Cognitive Processes in Translation and Interpreting. Critical Issues. In: DANKS, J.H.; SHREVE, G. M.; FOUNTAIN, S. B.; MCBEATH, M. K. (eds.), Cognitive Processes in Translation and Interpreting. Thousand Oaks Londres-Nueva Delhi: Sage, 1997.

STEFFENSEN, M. S.; JOAG-DEV, C. Cultural knowledge and reading. In ALDERSON, J. C.; URQUHART, A. H. (eds.). Reading in a foreign language. Nueva York: Longman, 1984.

STOCKWELL, P. Cognitive Poetics: An Introduction. Londres: Routledge, 2002.

SWAFFER, J. K. Headers, Texts, and Second Languages: The Interactive Processes. In: The Modem Language Journal, 72 (2), pp. 123-149, 1988.

TIRKKONEN-CONDIT, S.; LAUKKANEN, J. Evaluations - a key towardsunderstanding the affective dimension of translational decisions. Meta, XLI (1), pp. 45-59, 1996. 
TOURY, G. The Nature and Role of Norms in Translation. In: Descriptive Translation Studiesand beyond, pp. 53-69. Ámsterdam y Filadelfia: John Benjamins, 1995 [1978].

VAN DIJK, T.A. Tekstwetenschap. Een interdisciplinaire inleiding. [La ciencia del texto. Un enfoque interdisciplinario]. Ámsterdam: Het Spectrum, 1978.

VAN DIJK, T.A. Estructuras y funciones del discurso Trad. Myra Gann y Martí Mur. Bogotá: Siglo XXI, 1980.

VAN DIJK, T.A. Discourse and Knowledge: A Sociocognitive Approach. Cambridge: CUP, 2014.

VAN DIJK, T.A. y Kintsch, W. Strategies of Discourse Comprehension. San Diego: Academic Press, 1983.

VÁZQUEZ-AYORA, G. Introducción a la Traductología. Washington: Georgetown University Press, 1977.

VENUTTI, L. The Poet's Version; or, An Ethics of Translation. In: Translation Studies, v.4, n 2, pp. 230-247, 2011.

WAERN, Y. Thinking aloud during reading. A descriptive model and its application =Reports from the Department of Psychology, 546. Estocolmo: Stockholm University, Department of Psychology, 1979.

WILDE, S. (ed.). Notes from a kidwatcher: Selected writings of Yetta M. Goodman. Portsmouth: Heinemann, 1996.

WILliAM, J.; CHESTERMAN, A. The Map: A Beginner's Guide to Doing Research in Translation Studies. Oxon: Routledge, 2014.

WROE, N. Chinua Achebe: A life in writing. The Guardian. 13 de diciembre de 2010. Recuperado de: <https://www.theguardian.com/culture/2010/dec/13/chinua-achebe-life-inwriting>.

Recebido em: 15/09/2019

Aceito em: 07/11/2019 